\title{
Strategy for Curative Endoscopic Resection of Undifferentiated-Type Early Gastric Cancer
}

\author{
Jie-Hyun Kim \\ Division of Gastroenterology, Department of Internal Medicine, Gangnam Severance Hospital, Yonsei University College of Medicine, Seoul, \\ Korea \\ Endoscopic resection (ER) of undifferentiated-type early gastric cancer (UD-EGC) has a lower curative resection (CR) rate than \\ differentiated-type EGC. However, if UD-EGC is curatively resected using ER, long-term outcomes can be favorable. Thus, the strategy \\ for CR by ER is important in UD-EGC. To achieve CR in UD-EGC, biological behaviors including tumor growth patterns must be \\ considered. This review aims to describe what is important for curative ER of UD-EGC. Clin Endosc 2019;52:9-14
}

Key Words: Stomach neoplasms; Endoscopic mucosal resection; Histology

\section{INTRODUCTION}

Although endoscopic resection (ER) is a standard treatment for early gastric cancer (EGC) without lymph node metastasis (LNM), ER is performed more carefully in cases of undifferentiated-type EGC (UD-EGC) than in cases of differentiated-type EGC (D-EGC). This may be because the undifferentiated-type histology has been known to show aggressive biologic behavior in gastric cancer. According to a recent systematic review, the risk of LNM in UD-EGC cases that met the expanded criteria of ER was significantly increased compared with the risk in cases that met the absolute criteria. ${ }^{1}$ Nonetheless, many studies have reported the feasibility of ER in UD-EGC based on long-term follow-up outcome data. ${ }^{2-6}$ When curatively resected using ER, the long-term outcomes are favorable in UD-EGC. ${ }^{2-6}$ However, the curative resection

Received: November 1, 2018 Revised: January 2, 2019

Accepted: January 4, 2019

Correspondence: Jie-Hyun Kim

Division of Gastroenterology, Department of Internal Medicine, Gangnam Severance Hospital, Yonsei University College of Medicine, 211 Eonju-ro, Gangnamgu, Seoul 06273, Korea

Tel: +82-2-2019-3505, Fax: +82-2-3463-3882, E-mail: otilia94@yuhs.ac ORCID: https://orcid.org/0000-0002-9198-3326

cc This is an Open Access article distributed under the terms of the Creative Commons Attribution Non-Commercial License (http://creativecommons.org/ licenses/by-nc/3.0) which permits unrestricted non-commercial use, distribution, and reproduction in any medium, provided the original work is properly cited.
(CR) rate after ER is reportedly lower in cases of UD-EGC than in cases of D-EGC. ${ }^{2-4,6}$ This review aims to describe what is important for curative ER in UD-EGC.

\section{HISTOLOGIC DIAGNOSIS}

The histologic diagnosis is very important in the choice of treatment modality for EGC. In particular, discriminating between differentiated- and undifferentiated-type histology in EGC is important because the indications for ER differ. The situation becomes more complicated when the differentiated-type histology before ER changes to undifferentiated-type histology after ER. To achieve CR after ER in UD-EGC, it is important to identify undifferentiated-type histology before ER. Thus, several studies investigated the factors associated with UD-EGC exhibiting differentiated-type histology on biopsy. Moderately differentiated histology on biopsy, size $>2$ $\mathrm{cm}$, and body location were associated with UD-EGC exhibiting differentiated histology on biopsy. ${ }^{7-9}$ Tumor gross appearance can also be helpful for predicting the histologic findings. As the endoscopic elevated gross type is strongly associated with D-EGC, ${ }^{10,11}$ a recent study suggested that the presence of elevated-type EGC may exclude UD-EGC without need for a biopsy. ${ }^{10}$

Most of all, the accuracy of histologic diagnosis is import- 
ant. For target biopsy or histologic predictions, image-enhanced endoscopy or laser endomicroscopy can be helpful. A targeted biopsy based on confocal laser endomicroscopy found a higher proportion of cancer cells in biopsy samples with undifferentiated-type histology, including poorly differentiated adenocarcinoma (PD) and signet ring cell carcinoma (SRC), than that based on white-light endoscopy. ${ }^{12}$ On whitelight endoscopy, the actual biopsy site may be important according to a previous histopathological mapping study. ${ }^{7}$ UD-EGC cases that exhibited differentiated-type histology on biopsy frequently had a zone of transition from differentiatedto undifferentiated-type histology in ER specimens. ${ }^{7}$ The zone of transition occurred in one or two peripheral regions of the lesion. Therefore, a biopsy of several peripheral sites can be helpful in making an accurate diagnosis of UD-EGC before ER. $^{7}$

\section{DIFFERENCES WITHIN UD-EGC: PD VS. SRC}

ER is performed according to the Japanese histological classification of differentiated- and undifferentiated-type histology. According to a previous World Health Organization pathological classification, PD and SRC are undifferentiated-type histology types. The present indication for ER is the same as for undifferentiated-type histology, with no difference between PD and SRC. To date, there has been no evidence that different criteria should be applied in cases of PD vs. SRC. The long-term outcomes of ER reportedly do not differ between PD and SRC cases. ${ }^{3,6}$

Nonetheless, biological behaviors differ between PD and SRC. UD-EGC cases generally show higher frequencies of LNM than D-EGC cases; thus, the present criteria for ER are stricter in UD-EGC cases than in D-EGC cases. However, in EGC, SRC shows a better prognosis with less LNM than nonSRC. ${ }^{13-16}$ Growth patterns of cancer cells differ between PD and SRC, as known from their predominant gross appearance. The predominant gross appearance of EGC was the depressed type in PD, with a more infiltrative growth pattern of tumor cells, versus the flat type in SRC, with a spreading growth pattern of tumor cells. ${ }^{3,11,17}$ That is, cancer cells in PD have a vertical growth pattern, whereas those in SRC have a horizontal growth pattern. ${ }^{3,6,11,17}$ The different growth patterns of PD and SRC are reflected in the different results after ER. After $\mathrm{ER}$, the main cause of non-CR differed between PD and SRC, based on a positive vertical margin in $\mathrm{PD}$ vs. a positive lateral margin in SRC. ${ }^{3,6,17}$ Therefore, different strategies for PD and SRC are necessary to achieve CR after ER. For PD, accurate prediction of invasion depth is important, in contrast with that of tumor lateral extent for SRC, ${ }^{3,17}$

Further investigations are necessary to determine the optimal pre-ER evaluation. The most important step is evaluating whether LNM is present on computed tomography and/or endoscopic ultrasonography (EUS). Furthermore, the risk of LNM should be evaluated by examining invasion depth and tumor extent. However, the role of image-enhanced endoscopy and EUS in the accurate prediction of invasion depth or tumor extent should be investigated. There is currently no solid evidence for the routine application of EUS or image-enhanced endoscopy in pre-ER evaluation.

Considering vertical margin positive status after ER in PD, EUS with strict criteria may be helpful in accurately predicting invasion depth despite the possibility of underestimation using EUS in PD. ${ }^{18,19}$

To predict tumor extent in UD-EGC, chromoendoscopy and narrow-band imaging with magnifying endoscopy (NBI-ME) were not helpful, in contrast with their value in D-EGC. ${ }^{20,21}$ Thus, a Japanese algorithm recommended biopsies from the surrounding mucosa to delineate unclear tumor margins in UD-EGC, ${ }^{22}$ because SRC often shows a subepithelial spread pattern without superficial mucosal change. However, studies recently reported that NBI-ME can be helpful for accurately predicting tumor extent in UD-EGC. ${ }^{23-25}$ Based on the change in mucosal layer according to cancer cell infiltration, these studies categorized the findings of NBI-ME into three patterns: an extended intervening component pattern (tumor confined to the proliferative zone); a wavy microvessel pattern (tumor extending from the superficial layer to the proliferative zone); and a corkscrew pattern (tumor involving the entire mucosa). ${ }^{23-25}$ The corkscrew pattern is a well-known finding of NBI-ME in UD-EGC. However, this pattern can be found when tumor cells occupy the entire mucosa. The other two patterns have probably been missed by NBI-ME, which is why the role of NBI-ME has been limited in UD-EGC until now. To accurately predict tumor extent, NBI-ME can be helpful based on these three types, according to the sites occupied by tumor cells in SRC.

Tumor growth pattern may be predicted based on the surrounding mucosa in SRC. ${ }^{26}$ According to a previous report, the mucosa surrounding SRC may be an important mechanical barrier to tumor cell spread. Thus, SRC surrounded by atrophy or intestinal metaplasia may spread in a subepithelial manner. ${ }^{26}$ When SRC is surrounded by atrophy or intestinal metaplasia, tumor extent should be carefully delineated (Fig. 1). 

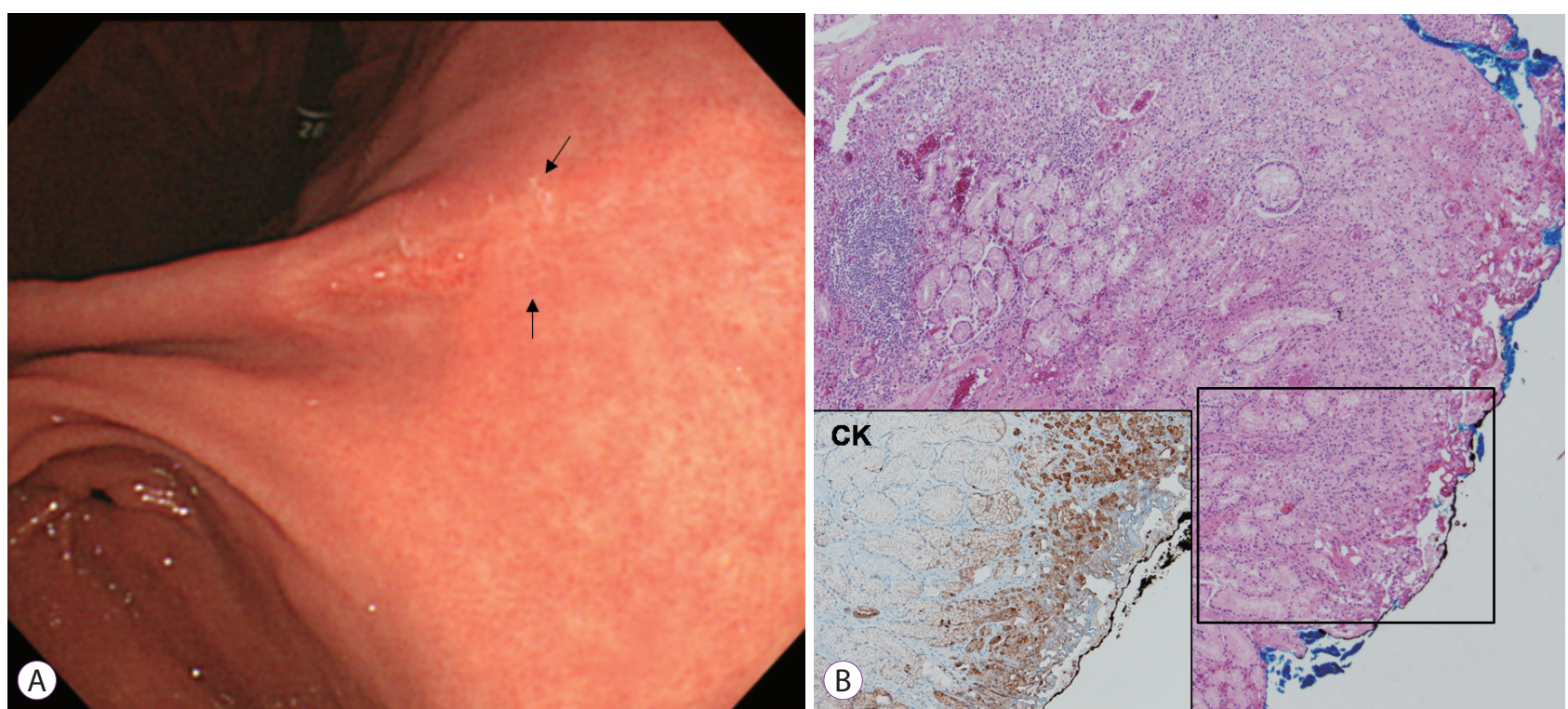

Fig. 1. Signet ring cell carcinoma (SRC) case with positive lateral margin after endoscopic resection. (A) Endoscopic image of early gastric cancer, showing a depressed lesion located in the posterior wall of the angle. The surrounding mucosa was combined with atrophic gastritis. After endoscopic resection, the lateral margin was positive (arrow). (B) Pathological findings after endoscopic resection (hematoxylin and eosin, $\times 40$ ). SRC cells showed subepithelial spread. Immunohistochemical staining for CK (AE1/AE3), $\times 100$.

\section{SITUATION AFTER ER: MIXED HISTOLOGY AND SAFETY MARGIN}

The histologic situation prior to ER is simplified when cases are classified as having differentiated- or undifferentiated-type histology. However, after ER, the histologic situation can become complex (e.g., mixed histology due to histologic heterogeneity within the tumor). The mixed type in the Lauren classification includes components of the intestinal type (50\%) and the diffuse type (50\%). However, most tumors have a primary histology with a minor histologic component $(<50 \%)$. The present ER criteria did not include mixed histology or minor histologic components.

Mixed histology, including undifferentiated-type histology as the primary or minor component, shows aggressive biologic behavior, compared with that in the non-mixed type. . $^{29}$ EGC with an SRC minor component showed a higher degree of LNM than cases without an SRC minor component. ${ }^{13}$ SRC is known to have less frequent LNM than PD; however, mixed SRC as a minor component can show more aggressive behavior than other histologies, including PD. ${ }^{13}$ Among D-EGC cases, lesions with a minor PD component showed higher frequencies of LNM than those without a PD component. ${ }^{29,30}$ In previous studies, a mixed histology in EGC was associated with a larger tumor size, submucosal invasion, more lymphovascular invasion, and higher LNM rates than in cases with a non-mixed histology. ${ }^{28,31-37}$ However, treatment outcomes after ER are inconsistent; some studies reported a higher non-CR rate and local recurrence, whereas some reported favorable long-term outcomes. ${ }^{33,35}$

Thus, are new ER criteria for mixed histology in EGC necessary? One study investigated LNM rates according to ER criteria in mixed histology cases. The study included SRC cases with mixed histology, mixed-type Lauren classification, and differentiated- or undifferentiated-predominant mixedtype as mixed histology cases. ${ }^{32}$ Lesions categorized as mixed histology in 3,419 EGC cases (49.7\% differentiated-type; 50.3\% undifferentiated-type) were investigated for LNM rates using the present ER criteria. The results showed that LNM was not found in lesions that met the present ER criteria. ${ }^{32}$

Another important factor in achieving CR is the pathological safety margin after ER. There is currently no definition of the ideal pathological safety margin after ER in EGC. Thus, complete resection after ER is defined as no cancer cell exposure to any resected margin with a line between normal tissue and the portion denatured by burning. ${ }^{17}$ To investigate the optimal pathological safety margin after ER, it is important to analyze risk factors for residual tumors in cases of completely resected EGC after ER. According to the study, a safety margin of $<3 \mathrm{~mm}$ (odds ratio [OR], 13.8), $\mathrm{PD}(\mathrm{OR}, 16.3)$, and SRC (OR, 9.8) were significantly associated with residual tumors after ER. ${ }^{38}$ That is, UD-EGC including both PD and SRC is an independent risk factor for the presence of residual tumor cells after negative resected margins are found in ER specimens. Furthermore, the pathological safety margin after ER may be $>3 \mathrm{~mm} .^{38}$ This safety margin can be important, espe- 
cially in cases of UD-EGC.

\section{STRATEGY FOR ACHIEVING CR USING ER IN UD-EGC}

Fig. 2 shows a suggested algorithm for achieving curative ER in cases of UD-EGC, based on the findings of previous studies. ${ }^{39}$

An accurate histologic diagnosis prior to ER is more important in UD-EGC than in D-EGC for CR after ER. Different tumor growth patterns should be considered for PD and SRC. For PD, the prediction of invasion depth can be import- ant, whereas for SRC, tumor extent is important. To predict tumor extent accurately in SRC, tumor cell involvement of the mucosal layer can be important. After ER, when a mixed histology is observed, the current ER criteria can be applied based on major histologic type. After CR by ER, if the pathological safety margin is $<3 \mathrm{~mm}$, short-term follow-up with biopsy at the resected base may be necessary to evaluate the risk of residual tumor development.

\section{CONCLUSIONS}

ER of UD-EGC has a lower CR rate than D-EGC. However,

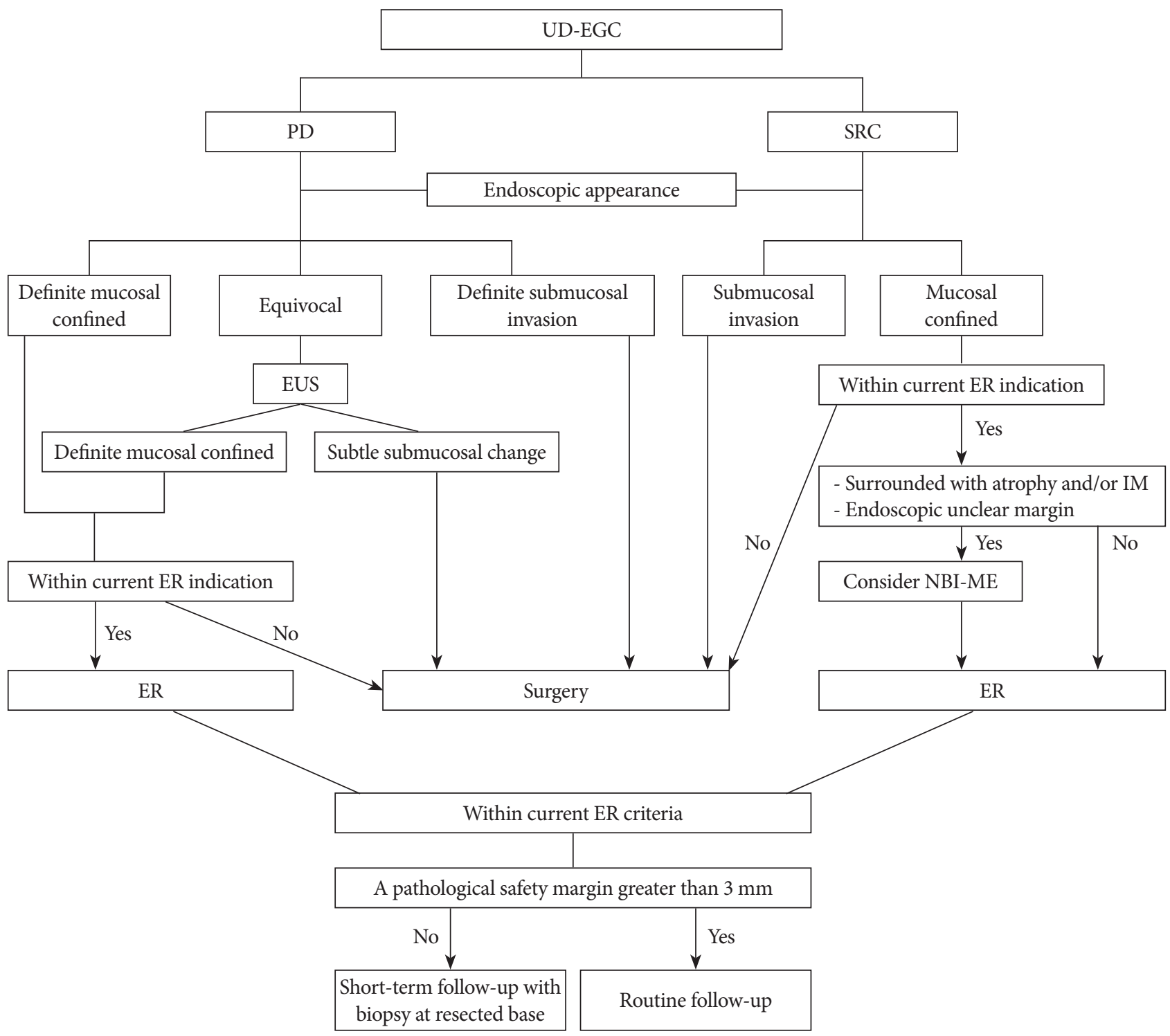

Fig. 2. Suggested algorithm for endoscopic resection (ER) of undifferentiated-type early gastric cancer (UD-EGC) based on previous studies (Modified from Kim ${ }^{39}$ ). PD, poorly differentiated adenocarcinoma; SRC, signet ring cell carcinoma; EUS, endoscopic ultrasonography; IM, intestinal metaplasia; NBI-ME, narrow-band imaging with magnifying endoscopy. 
if UD-EGC is curatively resected using ER based on the current criteria, long-term outcomes can be favorable. Thus, the strategy for CR by ER is important in UD-EGC. To achieve CR in UD-EGC, different biological behaviors, including tumor growth patterns between histologic types must be considered. Considering the growth patterns of cancer cells, prediction of invasion depth and lateral extent can be difficult in PD and SRC, respectively. Thus, advanced endoscopic tools including image-enhanced endoscopy or confocal laser endomicroscopy should be supplemented and developed to overcome these difficulties. After ER, the risk of residual tumor development should be carefully assessed in UD-EGC. A pathological safety margin $>3 \mathrm{~mm}$ may reduce the risk of residual tumor cells after ER.

\section{Conflicts of Interest}

The author has no financial conflicts of interest.

\section{REFERENCES}

1. Abdelfatah MM, Barakat M, Lee $\mathrm{H}$, et al. The incidence of lymph node metastasis in early gastric cancer according to the expanded criteria in comparison with the absolute criteria of the Japanese Gastric Cancer Association: a systematic review of the literature and meta-analysis. Gastrointest Endosc 2018;87:338-347.

2. Jeon HK, Lee SJ, Kim GH, Park DY, Lee BE, Song GA. Endoscopic submucosal dissection for undifferentiated-type early gastric cancer: shortand long-term outcomes. Surg Endosc 2018;32:1963-1970.

3. Kim JH, Kim YH, Jung DH, et al. Follow-up outcomes of endoscopic resection for early gastric cancer with undifferentiated histology. Surg Endosc 2014;28:2627-2633.

4. Ahn JY, Jung HY, Choi KD, et al. Endoscopic and oncologic outcomes after endoscopic resection for early gastric cancer: 1370 cases of absolute and extended indications. Gastrointest Endosc 2011;74:485-493.

5. Lee S, Choi KD, Han M, et al. Long-term outcomes of endoscopic submucosal dissection versus surgery in early gastric cancer meeting expanded indication including undifferentiated-type tumors: a criteria-based analysis. Gastric Cancer 2018;21:490-499.

6. Bang CS, Park JM, Baik GH, et al. Therapeutic outcomes of endoscopic resection of early gastric cancer with undifferentiated-type histology: a Korean ESD registry database analysis. Clin Endosc 2017;50:569-577.

7. Lee JH, Kim JH, Rhee K, et al. Undifferentiated early gastric cancer diagnosed as differentiated histology based on forceps biopsy. Pathol Res Pract 2013;209:314-318.

8. Min BH, Kang KJ, Lee JH, et al. Endoscopic resection for undifferentiated early gastric cancer: focusing on histologic discrepancies between forceps biopsy-based and endoscopic resection specimen-based diagnosis. Dig Dis Sci 2014;59:2536-2543.

9. Shim CN, Kim H, Kim DW, et al. Clinicopathologic factors and outcomes of histologic discrepancy between differentiated and undifferentiated types after endoscopic resection of early gastric cancer. Surg Endosc 2014;28:2097-2105.

10. Kanesaka T, Nagahama T, Uedo N, et al. Clinical predictors of histologic type of gastric cancer. Gastrointest Endosc 2018;87:1014-1022.

11. Jung DH, Park YM, Kim JH, et al. Clinical implication of endoscopic gross appearance in early gastric cancer: revisited. Surg Endosc 2013;27:3690-3695.

12. Park $\mathrm{CH}$, Kim H, Jo JH, et al. Role of probe-based confocal laser en- domicroscopy-targeted biopsy in the molecular and histopathological study of gastric cancer. J Gastroenterol Hepatol 2019;34:84-91.

13. Huh CW, Jung DH, Kim JH, et al. Signet ring cell mixed histology may show more aggressive behavior than other histologies in early gastric cancer. J Surg Oncol 2013;107:124-129.

14. Chiu CT, Kuo CJ, Yeh TS, et al. Early signet ring cell gastric cancer. Dig Dis Sci 2011;56:1749-1756.

15. Zhang M, Zhu G, Zhang H, Gao H, Xue Y. Clinicopathologic features of gastric carcinoma with signet ring cell histology. J Gastrointest Surg 2010;14:601-606.

16. Hyung WJ, Noh SH, Lee JH, et al. Early gastric carcinoma with signet ring cell histology. Cancer 2002;94:78-83.

17. Kim JH, Lee YC, Kim H, et al. Endoscopic resection for undifferentiated early gastric cancer. Gastrointest Endosc 2009;69:e1-e9.

18. Kim JH, Song KS, Youn YH, et al. Clinicopathologic factors influence accurate endosonographic assessment for early gastric cancer. Gastrointest Endosc 2007;66:901-908.

19. Choi J, Kim SG, Im JP, Kim JS, Jung HC, Song IS. Comparison of endoscopic ultrasonography and conventional endoscopy for prediction of depth of tumor invasion in early gastric cancer. Endoscopy 2010;42:705713 .

20. Lee BE, Kim GH, Park DY, et al. Acetic acid-indigo carmine chromoendoscopy for delineating early gastric cancers: its usefulness according to histological type. BMC Gastroenterol 2010;10:97.

21. Nagahama T, Yao K, Maki S, et al. Usefulness of magnifying endoscopy with narrow-band imaging for determining the horizontal extent of early gastric cancer when there is an unclear margin by chromoendoscopy (with video). Gastrointest Endosc 2011;74:1259-1267.

22. Yao K, Nagahama T, Matsui T, Iwashita A. Detection and characterization of early gastric cancer for curative endoscopic submucosal dissection. Dig Endosc 2013;25 Suppl 1:44-54.

23. Okada K, Fujisaki J, Kasuga A, et al. Diagnosis of undifferentiated type early gastric cancers by magnification endoscopy with narrow-band imaging. J Gastroenterol Hepatol 2011;26:1262-1269.

24. Horiuchi Y, Fujisaki J, Yamamoto N, et al. Accuracy of diagnostic demarcation of undifferentiated-type early gastric cancers for magnifying endoscopy with narrow-band imaging: endoscopic submucosal dissection cases. Gastric Cancer 2016;19:515-523.

25. Horiuchi Y, Fujisaki J, Yamamoto N, et al. Accuracy of diagnostic demarcation of undifferentiated-type early gastric cancer for magnifying endoscopy with narrow-band imaging: surgical cases. Surg Endosc 2017;31:1906-1913.

26. Kim H, Kim JH, Lee YC, et al. Growth patterns of signet ring cell carcinoma of the stomach for endoscopic resection. Gut Liver 2015;9:720726.

27. Zheng HC, Li XH, Hara T, et al. Mixed-type gastric carcinomas exhibit more aggressive features and indicate the histogenesis of carcinomas. Virchows Arch 2008;452:525-534.

28. Lee JH, Choi IJ, Han HS, et al. Risk of lymph node metastasis in differentiated type mucosal early gastric cancer mixed with minor undifferentiated type histology. Ann Surg Oncol 2015;22:1813-1819.

29. Jung DH, Bae YS, Yoon SO, et al. Poorly differentiated carcinoma component in submucosal layer should be considered as an additional criterion for curative endoscopic resection of early gastric cancer. Ann Surg Oncol 2015;22 Suppl 3:S772-S777.

30. Horiuchi Y, Fujisaki J, Yamamoto N, et al. Undifferentiated-type component mixed with differentiated-type early gastric cancer is a significant risk factor for endoscopic non-curative resection. Dig Endosc 2018;30:624-632.

31. Pyo JH, Lee H, Min BH, et al. Early gastric cancer with a mixed-type Lauren classification is more aggressive and exhibits greater lymph node metastasis. J Gastroenterol 2017;52:594-601.

32. Yoon HJ, Kim YH, Kim JH, et al. Are new criteria for mixed histology necessary for endoscopic resection in early gastric cancer? Pathol Res Pract 2016;212:410-414. 
33. Min BH, Kim KM, Park CK, et al. Outcomes of endoscopic submucosal dissection for differentiated-type early gastric cancer with histological heterogeneity. Gastric Cancer 2015;18:618-626.

34. Shim CN, Chung H, Park JC, et al. Early gastric cancer with mixed histology predominantly of differentiated type is a distinct subtype with different therapeutic outcomes of endoscopic resection. Surg Endosc 2015;29:1787-1794

35. Han JP, Hong SJ, Kim HK. Long-term outcomes of early gastric cancer diagnosed as mixed adenocarcinoma after endoscopic submucosal dissection. J Gastroenterol Hepatol 2015;30:316-320.

36. Takizawa K, Ono H, Kakushima N, et al. Risk of lymph node metastases from intramucosal gastric cancer in relation to histological types: how to manage the mixed histological type for endoscopic submucosal dissection. Gastric Cancer 2013;16:531-536.

37. Hanaoka N, Tanabe S, Mikami T, Okayasu I, Saigenji K. Mixed-histologic-type submucosal invasive gastric cancer as a risk factor for lymph node metastasis: feasibility of endoscopic submucosal dissection. Endoscopy 2009;41:427-432.

38. Yun GW, Kim JH, Lee YC, et al. What are the risk factors for residual tumor cells after endoscopic complete resection in gastric epithelial neoplasia? Surg Endosc 2015;29:487-492.

39. Kim JH. Important considerations when contemplating endoscopic resection of undifferentiated-type early gastric cancer. World J Gastroenterol 2016;22:1172-1178. 